\title{
Temperature-dependent vortex motion in a square mesoscopic superconducting cylinder: Ginzburg-Landau calculations
}

\author{
Edson Sardella, ${ }^{*}$ André Luiz Malvezzi ${ }^{\dagger}$ and Paulo Noronha Lisboa-Filho \\ Departamento de Física, Faculdade de Ciências, Universidade Estadual Paulista-UNESP, Caixa Postal 473, 17033-360, Bauru-SP, \\ Brazil \\ Wilson Aires Ortiz \\ Departamento de Física, Universidade Federal de São Carlos-UFSCar, Caixa Postal 676, 13566-905, São Carlos-SP, Brazil
}

(Received 5 May 2006; published 21 July 2006)

\begin{abstract}
In this work we investigate the dynamics of vortices in a square mesoscopic superconductor. As time evolves we show how the vortices are nucleated into the sample to form a multivortex, single vortex, and giant vortex states. We illustrate how the vortices move around at the transition fields before they accommodate into an equilibrium configuration. We also calculate the magnetization and the free energy as functions of the applied magnetic field for several values of temperature. In addition, we evaluate the upper critical field.
\end{abstract}

DOI: 10.1103/PhysRevB.74.014512

PACS number(s): 74.25.-q, 74.20.De, 74.78.Na

\section{INTRODUCTION}

The study of superconducting samples in mesoscopic scale presented a remarkable improvement during the last years. Certainly, such interest is based on the fact that when the size of the samples is close to the order of the coherence length $\xi$ and/or the size of the penetration depth $\lambda$, there are some significant modifications on the physical properties of the superconducting state. Among such differences, the occurrence and dynamics of vortices have been extensively reported in the literature. ${ }^{1,2}$ Besides size effects, the geometrical configuration of the samples also plays a significant role. ${ }^{1}$ Taking into account vortex-vortex interaction and boundary effects, two different types of vortex configurations can be achieved in mesoscopic samples. One of them is the multivortex state (MVS) characterized by the existence of several single quantized vortices. The other possibility is the socalled giant vortex state (GVS) where more than one quantum of flux can be measured.

In terms of the theoretical prediction of GVS and the collapse of MVS into GVS, several studies reported not just the occurrence of GVS, but also a multiple form of a combination of these states. ${ }^{1,3}$ However, the direct experimental observation of GVS and MVS-GVS (coexistence of both states) is still controversial. ${ }^{4-6}$

One of the most used approaches to study mesoscopic superconductors is the time dependent Ginzburg-Landau equations (TDGL). This theory has been successfully applied for thin square mesoscopic superconductors. For instance, Mel'nikov et $a l .{ }^{7}$ have used the TDGL equations to study the vortex configurations close to the upper critical field in such systems. Otherwise, Hernandez et al. ${ }^{8}$ focused on the effects of thermal fluctuations in the magnetization and free energy as function of the external applied magnetic field.

In the present contribution, we study the time evolution of the superconducting state of a long cylinder of square cross section by slowly increasing the applied external field. Despite our results show some subtle differences compared with recent work on thin mesoscopic squares ${ }^{1}$ concerning the formation of GVS, MVS, and MVS-GVS, most of them strengthen the conclusions of the existence of such vortex configurations. We must stress that the emphasis of our work held on the description of the vortex dynamics for different temperatures in a condition of infinitesimal growing applied external field.

The paper is outlined as follows. In Sec. II we present the theoretical approach which will be used. Our description of the superconducting state is based on the time dependent Ginzburg-Landau equations. We also present the main steps of the $\psi-U$ method used to solve the TDGL equations numerically. In Sec. III, the upper critical field, the magnetization, and energy curves are shown and discussed. In addition, we shall discuss the output of the TDGL equations concerning the GVS, MVS, and MVS-GVS.

\section{THE THEORETICAL MODEL AND THE NUMERICAL SETUP}

We investigate the dynamics of the vortices for a long and thin superconducting cylinder of square cross section. For the sake of brevity, from now on we will refer to this geometry as a square superconductor. Thus, we are allowed to take the order parameter and the local magnetic field invariant along the $z$ direction. The vortex configurations at the equilibrium can be obtained from the Ginzburg-Landau (GL) equations of superconductivity. Schmid ${ }^{9}$ generalized the GL equations which account for the time evolution of the vortices until the most stable configuration is accomplished. The time evolution was incorporated to the GL equations in such a manner that their gauge invariance is preserved. The TDGL equations for the complex order parameter $\psi$, the vector potential $\mathbf{A}$, and the scalar electrical potential $\Phi$ are given by

$$
\begin{gathered}
\frac{\hbar^{2}}{4 m D}\left(\frac{\partial}{\partial t}+\frac{i e}{\hbar} \Phi\right) \psi=-\frac{1}{4 m}\left(-i \hbar \nabla-\frac{2 e}{c} \mathbf{A}\right)^{2} \psi+a \psi-b|\psi|^{2} \psi \\
\nu\left(\frac{1}{c} \frac{\partial \mathbf{A}}{\partial t}+\nabla \Phi\right)=\mathbf{J}_{s}-\frac{c}{4 \pi} \nabla \times \mathbf{A}
\end{gathered}
$$

where the supercurrent density is 


$$
\mathbf{J}_{s}=\frac{e}{m} \mathfrak{R}\left[\bar{\psi}\left(-i \hbar \nabla-\frac{2 e}{c} \mathbf{A}\right) \psi\right],
$$

where $\mathfrak{R}$ indicates the real part and the overbar the complex conjugation. Here, $D$ is the diffusion coefficient, $\nu$ is the electrical conductivity, and $a$ and $b$ are two positive phenomenological constants; we use the temperature dependence $a$ $=a(T)=a_{0}\left(T_{c}-T\right)$, for all temperatures $T \leqslant T_{c}$, where $T_{c}$ is the critical temperature. The quantity $|\psi|^{2}$ represents the local density of Cooper pairs and the local magnetic field is given by $\mathbf{h}=\boldsymbol{\nabla} \times \mathbf{A}$. We consider the zero-electrical potential gauge at all times, $\Phi=0$.

We solve the TDGL subject to the following boundary conditions: $\left.(-i \boldsymbol{\nabla}-\mathbf{A})\right|_{n}=0$ normal to the sample surfaces, and $h_{z}=H$ parallel to the sample surfaces, where $H$ is the external field uniformly applied along the $z$ direction.

It is convenient to write the above equations in dimensionless units. We use the following units for the order parameter, length, time, and magnetic field, respectively:

$$
\begin{gathered}
\psi_{\infty}^{2}=\frac{a}{b}=\frac{a_{0} T_{c}}{b}\left(1-T / T_{c}\right), \\
\xi^{2}(0)=\frac{\hbar^{2}}{4 m a_{0} T_{c}}, \\
\tau=\frac{\xi^{2}(0)}{D}, \\
H_{c 2}(0)=\frac{\Phi_{0}}{2 \pi \xi^{2}(0)},
\end{gathered}
$$

and $T$ in units of $T_{c}$.

Then, the nondimensional TDGL equations can be rewritten as

$$
\begin{gathered}
\frac{\partial \psi}{\partial t}=-(-i \boldsymbol{\nabla}-\mathbf{A})^{2} \psi+(1-T) \psi\left(1-|\psi|^{2}\right), \\
\beta \frac{\partial \mathbf{A}}{\partial t}=\mathbf{J}_{s}-\kappa^{2} \boldsymbol{\nabla} \times \mathbf{A}, \\
\mathbf{J}_{s}=(1-T) \Re[\bar{\psi}(-i \boldsymbol{\nabla}-\mathbf{A}) \psi],
\end{gathered}
$$

where $\kappa=\lambda(T) / \xi(T)=\lambda(0) / \xi(0)$ is the GL parameter and $\beta$ $=4 \pi \nu D / c^{2} \xi^{2}(0)$; here

$$
\begin{gathered}
\lambda(T)=\left(\frac{m c^{2} b}{8 \pi e^{2} a(T)}\right)^{1 / 2}, \\
\xi(T)=\left(\frac{\hbar^{2}}{4 m a(T)}\right)^{1 / 2},
\end{gathered}
$$

are the London penetration length and the coherence length, respectively.

In order to discretize the TDGL equations we use the $\psi-U$ method. ${ }^{10}$ This method has the important characteristic of preserving the gauge invariance of the TDGL equations.
This is accomplished by introducing the auxiliary fields

$$
\begin{aligned}
& U_{x}(x, y, t)=\exp \left(-i \int_{x_{0}}^{x} A_{x}(s, y, t) d s\right), \\
& U_{y}(x, y, t)=\exp \left(-i \int_{y_{0}}^{y} A_{y}(x, s, t) d s\right),
\end{aligned}
$$

where $\left(x_{0}, y_{0}\right)$ is an arbitrary point.

Thus, the first of the TDGL equations and the supercurrent density can be written in the following form:

$$
\begin{gathered}
\frac{\partial \psi}{\partial t}=U_{x} \frac{\partial^{2}\left(U_{x} \psi\right)}{\partial x^{2}}+U_{y} \frac{\partial^{2}\left(U_{y} \psi\right)}{\partial y^{2}}+(1-T) \psi\left(1-|\psi|^{2}\right) \\
J_{s \alpha}=\frac{(1-T)}{2 i}\left[U_{\alpha} \bar{\psi} \frac{\partial\left(U_{\alpha} \psi\right)}{\partial \alpha}-U_{\alpha} \psi \frac{\partial\left(U_{\alpha} \bar{\psi}\right)}{\partial \alpha}\right] \\
=(1-T) \mathfrak{I}\left[U_{\alpha} \bar{\psi} \frac{\partial\left(U_{\alpha} \psi\right)}{\partial \alpha}\right]
\end{gathered}
$$

where $\alpha=(x, y)$, and $\mathfrak{I}$ indicates the imaginary part.

The full discretization of the TDGL equations can be found in more detail in Refs. 10 and 11 Here, we only show the results. The discretizations are carried out in a rectangular mesh consisting of $N_{x} \times N_{y}$ cell points. We denote by $\Delta t$ the incremental time, by $\left(a_{x}, a_{y}\right)$ the lattice spacing between adjacent cell points, and by $\left(x_{i}, y_{j}\right)$ an arbitrary vertex point in the mesh. Let us define

$$
\begin{gathered}
t_{n}=n \Delta t, \quad n=0,1,2,3, \ldots, \\
x_{i}=(i-1) a_{x}, \quad i=1,2,3, \ldots, N_{x}+1, \\
y_{j}=(j-1) a_{y}, \quad j=1,2,3, \ldots, N_{y}+1, \\
\psi_{i, j}^{n}=\psi\left(x_{i}, y_{j}, t_{n}\right), \\
U_{x, i, j}^{n}=U_{x}\left(x_{i+1}, y_{j}, t_{n}\right) \bar{U}_{x}\left(x_{i}, y_{j}, t_{n}\right)=\exp \left(-i \int_{x_{i}}^{x_{i+1}} A_{x}(s, y, t) d s\right), \\
U_{y, i, j}^{n}=U_{y}\left(x_{i}, y_{j+1}, t_{n}\right) \bar{U}_{y}\left(x_{i}, y_{j}, t_{n}\right)=\exp \left(-i \int_{y_{i}}^{y_{i+1}} A_{y}(x, s, t) d s\right), \\
h_{z, i, j}^{n}=h_{z}\left(x_{i}+\frac{a_{x}}{2}, y_{j}+\frac{a_{y}}{2}, t_{n}\right) .
\end{gathered}
$$

According to these definitions, it can be easily seen that $\psi$ is a vertex variable, $h_{z}$ is a cell variable, and $U$ is a link variable.

Then, the discretized version of the TDGL equations are given by 


$$
\begin{aligned}
\psi_{i, j}^{n+1}= & \psi_{i, j}^{n}+\Delta t \frac{U_{x, i, j}^{n} \psi_{i+1, j}^{n}-2 \psi_{i, j}^{n}+\bar{U}_{x, i-1, j}^{n} \psi_{i-1, j}^{n}}{a_{x}^{2}} \\
& +\Delta t \frac{U_{y, i, j}^{n} \psi_{i, j+1}^{n}-2 \psi_{i, j}^{n}+\bar{U}_{y, i, j-1}^{n} \psi_{i, j-1}^{n}}{a_{y}^{2}} \\
& +\Delta t(1-T) \psi_{i, j}^{n}\left(1-\left|\psi_{i, j}^{n}\right|^{2}\right), \\
U_{x, i, j}^{n+1}= & U_{x, i, j}^{n}+\Delta t \frac{(1-T)}{i \beta} U_{x, i, j}^{n} \Im\left[\bar{\psi}_{i, j}^{n} U_{x, i, j}^{n} \psi_{i+1, j}^{n}\right] \\
& -\Delta t \frac{\kappa^{2}}{\beta a_{y}^{2}} U_{x, i, j}^{n}\left(\bar{L}_{i, j-1}^{n} L_{i, j}^{n}-1\right), \\
U_{y, i, j}^{n+1}= & U_{y, i, j}^{n}+\Delta t \frac{(1-T)}{i \beta} U_{y, i, j}^{n} \Im\left[\bar{\psi}_{i, j}^{n} U_{y, i, j}^{n} \psi_{i, j+1}^{n}\right] \\
& -\Delta t \frac{\kappa^{2}}{\beta a_{x}^{2}} U_{y, i, j}^{n}\left(\bar{L}_{i, j}^{n} L_{i-1, j}^{n}-1\right),
\end{aligned}
$$

where

$$
L_{i, j}^{n}=U_{x, i, j}^{n} \bar{U}_{x, i, j+1}^{n} U_{y, i+1, j}^{n} \bar{U}_{y, i, j}^{n}=\exp \left(-i a_{x} a_{y} h_{z, i, j}^{n}\right) .
$$

The discretized boundary conditions are

$$
\begin{aligned}
& \psi_{1, j}^{n}=U_{x, 1, j}^{n} \psi_{2, j}^{n}, \quad \psi_{N_{x}+1, j}^{n}=\bar{U}_{x, N_{x}, j}^{n} \psi_{N_{x}, j}^{n}, \\
& \psi_{i, 1}^{n}=U_{y, i, 1}^{n} \psi_{i, 2}^{n}, \quad \psi_{i, N_{y}+1}^{n}=\bar{U}_{y, i, N_{y}}^{n} \psi_{i, N_{y}}^{n} .
\end{aligned}
$$

The auxiliary fields $U$ and $L$ are updated at any $(i, j)$ surface cell point by using the boundary condition $L_{i, j}^{n}$ $=\exp \left(-i a_{x} a_{y} H\right)$, whatever the instant $n$.

Starting from some initial conditions for $\psi$ and the auxiliary fields $U$, and upon using the TDGL Eqs. (9) and the boundary conditions (11), the new order parameter and the local magnetic field are determined. This process is repeated until a stationary state is achieved. Our procedure consists of starting from a certain value of $H$ and then we ramp up the applied external field in small intervals of $\Delta H=10^{-3}$. The stationary state found for a fixed value of $H$ is then used as the initial condition for $H+\Delta H$. For the first value of $H$, the system is initialized at the Meissner state where $\psi_{i, j}^{0}=1$, $U_{x, i, j}^{0}=1, U_{y, i, j}^{0}=1$. We must emphasize that no a priori vortex configuration is assumed to determine $(\psi, \mathbf{A}, \mathbf{h})$. In other words, the applied magnetic field is increased slowly from zero up to the upper critical field where the superconductivity is completely destroyed. At each fixed applied field we follow the time evolution of the local magnetic field and the superconducting order parameter until we obtain a steady state solution. Then, this stationary solution is used as the initial condition for the next applied field. Thus, as the applied magnetic field increases and the time changes, we preserve the magnetic history of the system. That is, we study the time evolution of the system at each fixed applied magnetic field by assuming that there is already some penetrated magnetic flux inside the sample.

The Gibbs free energy and the magnetization are very sensitive to changes in the vortex configurations. So, any physical relevant phenomenon should manifest into these quantities. The TDGL equations describe the gradient flow for the Gibbs free energy. Thus, in principle, the output of the TDGL should correspond to the global minimum of the energy of the system. The energy, in units of $\mathcal{G}_{0}=a_{0}^{2} T_{c}^{2} / b$, is given by

$$
\begin{aligned}
\mathcal{G}= & \frac{1}{V} \int\left[(1-T)|(-i \boldsymbol{\nabla}-\mathbf{A}) \psi|^{2}+(1-T)^{2}|\psi|^{2}\left(\frac{1}{2}|\psi|^{2}-1\right)\right. \\
& \left.+\kappa^{2}\left((\boldsymbol{\nabla} \times \mathbf{A})_{z}-H\right)^{2}\right] d V
\end{aligned}
$$

The discrete version of this equations is

$$
\begin{aligned}
\mathcal{G}_{n}= & \frac{1}{N_{x} N_{y}} \sum_{i=1}^{N_{x}} \sum_{j=1}^{N_{y}}\left\{\frac { ( 1 - T ) } { 2 } \left[\frac{\left|U_{x, i, j}^{n} \psi_{i+1, j}^{n}-\psi_{i, j}^{n}\right|^{2}}{a_{x}^{2}}\right.\right. \\
& +\frac{\left|U_{x, i, j+1}^{n} \psi_{i+1, j+1}^{n}-\psi_{i, j+1}^{n}\right|^{2}}{a_{x}^{2}}+\frac{\left|U_{y, i, j}^{n} \psi_{i, j+1}^{n}-\psi_{i, j}^{n}\right|^{2}}{a_{y}^{2}} \\
& \left.+\frac{\left|U_{y, i+1, j}^{n} \psi_{i+1, j+1}^{n}-\psi_{i+1, j}^{n}\right|^{2}}{a_{y}^{2}}\right]+\frac{(1-T)^{2}}{4}\left[| \psi _ { i , j } ^ { n } | ^ { 2 } \left(\frac{1}{2}\left|\psi_{i, j}^{n}\right|^{2}\right.\right. \\
& -1)+\left|\psi_{i+1, j}^{n}\right|^{2}\left(\frac{1}{2}\left|\psi_{i+1, j}^{n}\right|^{2}-1\right)+\left|\psi_{i+1, j+1}^{n}\right|^{2}\left(\frac{1}{2}\left|\psi_{i+1, j+1}^{n}\right|^{2}\right. \\
& \left.\left.-1)+\left|\psi_{i, j+1}^{n}\right|^{2}\left(\frac{1}{2}\left|\psi_{i, j+1}^{n}\right|^{2}-1\right)\right]+\left(h_{z, i, j}^{n}-H\right)^{2}\right\} .
\end{aligned}
$$

The magnetization $4 \pi M=B-H$, where $B$ is the induction (the spatial average of the local magnetic field), is

$$
-4 \pi M_{n}=\frac{1}{N_{x} N_{y}} \sum_{i=1}^{N_{x}} \sum_{j=1}^{N_{y}} h_{z, i, j}^{n}-H .
$$

We calculate the energy and the magnetization at each instant $n$, although we are most interested in their values at the stationary state. The stationary state is accepted when the absolute value of the order parameter does not change within

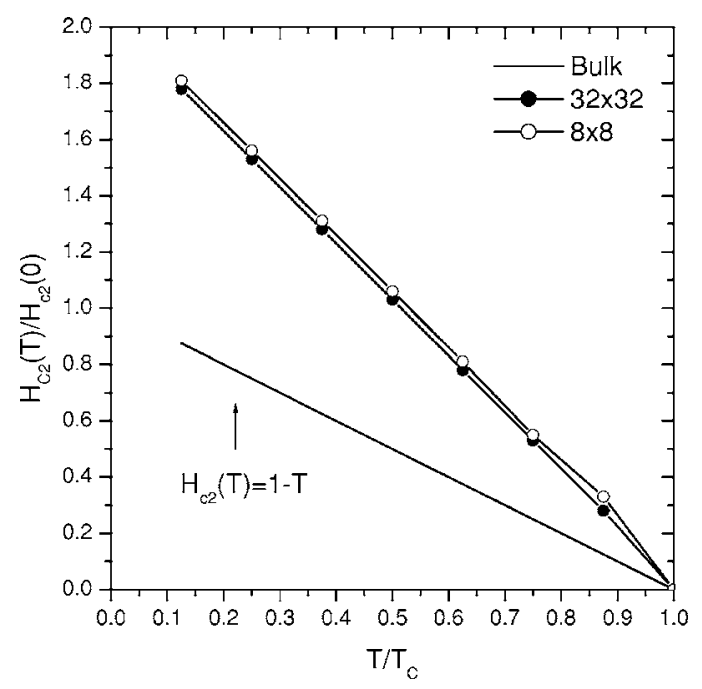

FIG. 1. The upper critical field for two superconducting square: $S_{1}$ (open circles); $S_{2}$ (filled circles), and bulk (solid straight line). 


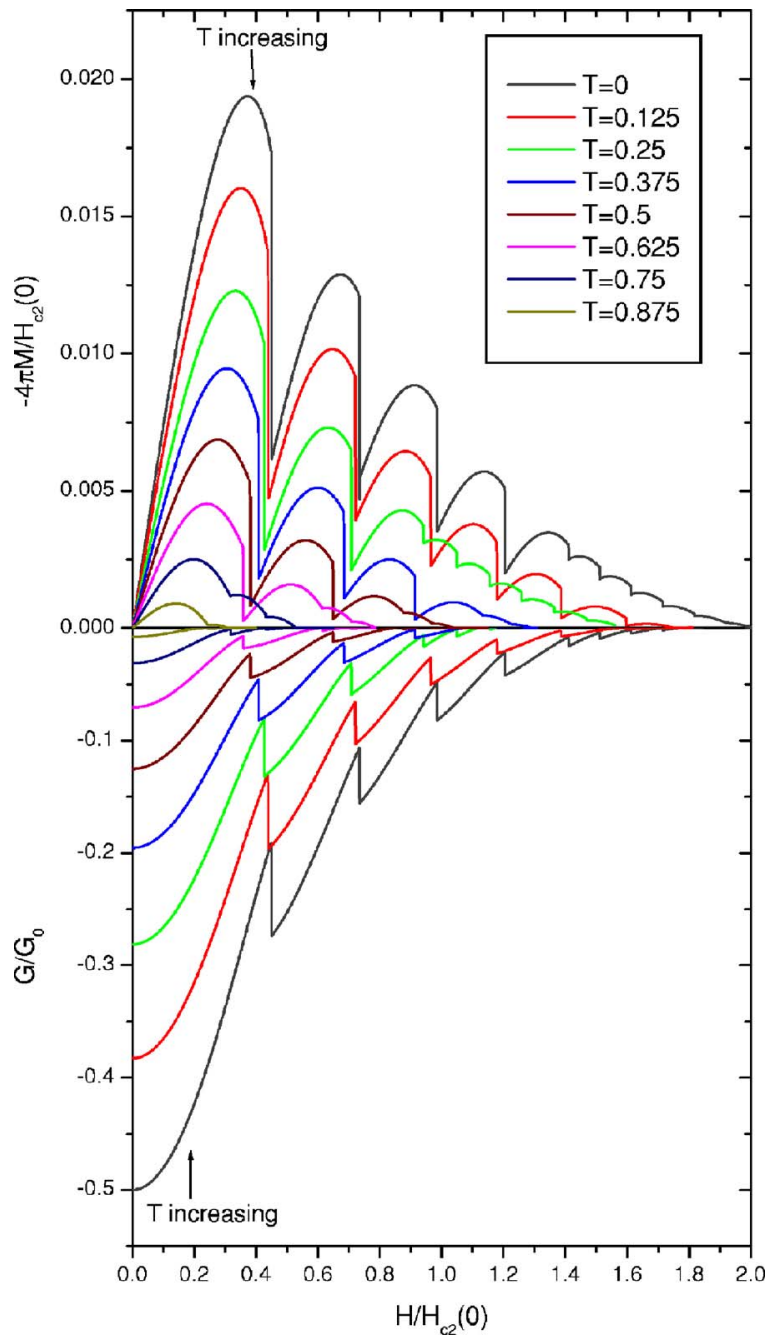

FIG. 2. (Color online) The magnetization (upper graphics) and the energy (lower graphics) as functions of the applied external applied field for several values of temperature for the $S_{1}$ superconductor.

a certain precision. The highest difference $\left\|\psi_{i, j}^{n+1}|-| \psi_{i, j}^{n}\right\|$ we allowed was $10^{-5}$ over a few thousand time steps.

For each fixed value of field and instant $n$, the output data of Eqs. (9)-(11) were stored in matrices and visualized in order to observe the dynamics of the vortices. The visualization process allows us to see how many vortices are nucleating inside the sample as the applied magnetic field increases. However, a more reliable manner of counting the number of vortices can be found by integrating Eq. (4) along a rectangle containing the superconducting square. This leads us to

$$
\oint \frac{1}{|\psi|^{2}} \mathfrak{R}[\bar{\psi}(-i \boldsymbol{\nabla}-\mathbf{A}) \psi] \cdot d \mathbf{r}=(2 \pi N-\Phi),
$$

where $N$ is the number of vortices (vorticity) and $\Phi$ is the total penetrated flux. The discrete counterpart of this equation is

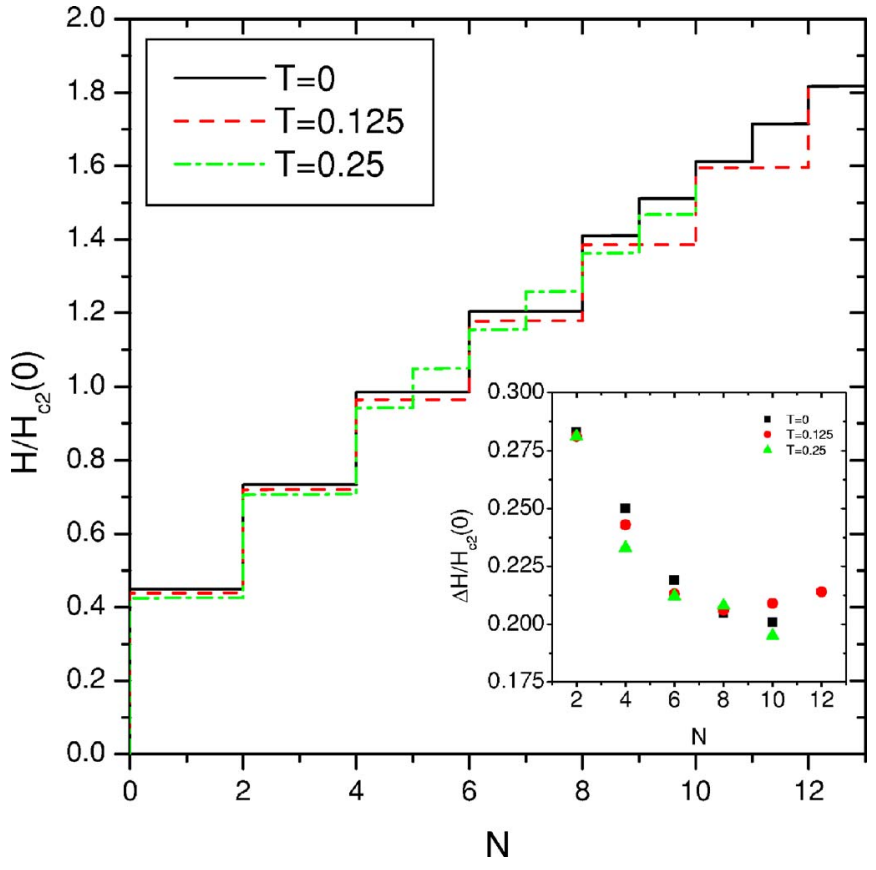

FIG. 3. (Color online) The applied magnetic field as a function of the number of vortices for three different temperatures. Inset: the values of applied magnetic field sufficient to change the vorticity from $N$ to $N+2$, with $N$ even.

$$
\begin{aligned}
N_{n}= & \frac{\Phi_{n}}{2 \pi}+\frac{1}{2 \pi} \sum_{i=1}^{N_{x}}\left\{\frac{1}{\left|\Psi_{i, 1}^{n}\right|^{2}} \Im\left[\bar{\psi}_{i, 1}^{n} U_{x, i, 1}^{n} \psi_{i+1,1}^{n}\right]\right. \\
& \left.-\frac{1}{\left|\Psi_{i, N_{y}+1}^{n}\right|^{2}} \Im\left[\bar{\psi}_{i, N_{y}+1}^{n} U_{x, i, N_{y}+1}^{n} \psi_{i+1, N_{y}+1}^{n}\right]\right\} \\
& +\frac{1}{2 \pi} \sum_{j=1}^{N_{y}}\left\{\frac{1}{\left|\Psi_{N_{x}+1, j}^{n}\right|^{2}} \Im\left[\bar{\psi}_{N_{x}+1, j}^{n} U_{y, N_{x}+1, j}^{n} \psi_{N_{x}+1, j+1}^{n}\right]\right. \\
& \left.-\frac{1}{\left|\Psi_{1, j}^{n}\right|^{2}} \Im\left[\bar{\psi}_{1, j}^{n} U_{y, 1, j}^{n} \psi_{1, j+1}^{n}\right]\right\},
\end{aligned}
$$

where, for instance,

$$
\left|\Psi_{i, 1}^{n}\right|^{2}=\frac{1}{2}\left(\left|\psi_{i, 1}^{n}\right|^{2}+\left|\psi_{i+1,1}^{n}\right|^{2}\right) .
$$

Notice that (16) has not been derived in Refs . 10 and 11.

\section{RESULTS AND DISCUSSION}

In order to carry out the numerical simulations we have chosen two superconducting squares of surface areas $S_{1}=8$ $\times 8$ and $S_{2}=32 \times 32$. The Ginzburg-Landau parameter used for both samples was $\kappa=5$, and $\beta=1$. Since the core size of the vortices diminishes as the temperature is lowered we have used two different meshes. For $T \geqslant 0.25$, a mesh with $32 \times 32$ cell points was sufficient to describe the detailed configuration of the vortices. Nevertheless, for lower temperatures we had to increase the number of cell points to $64 \times 64$. 

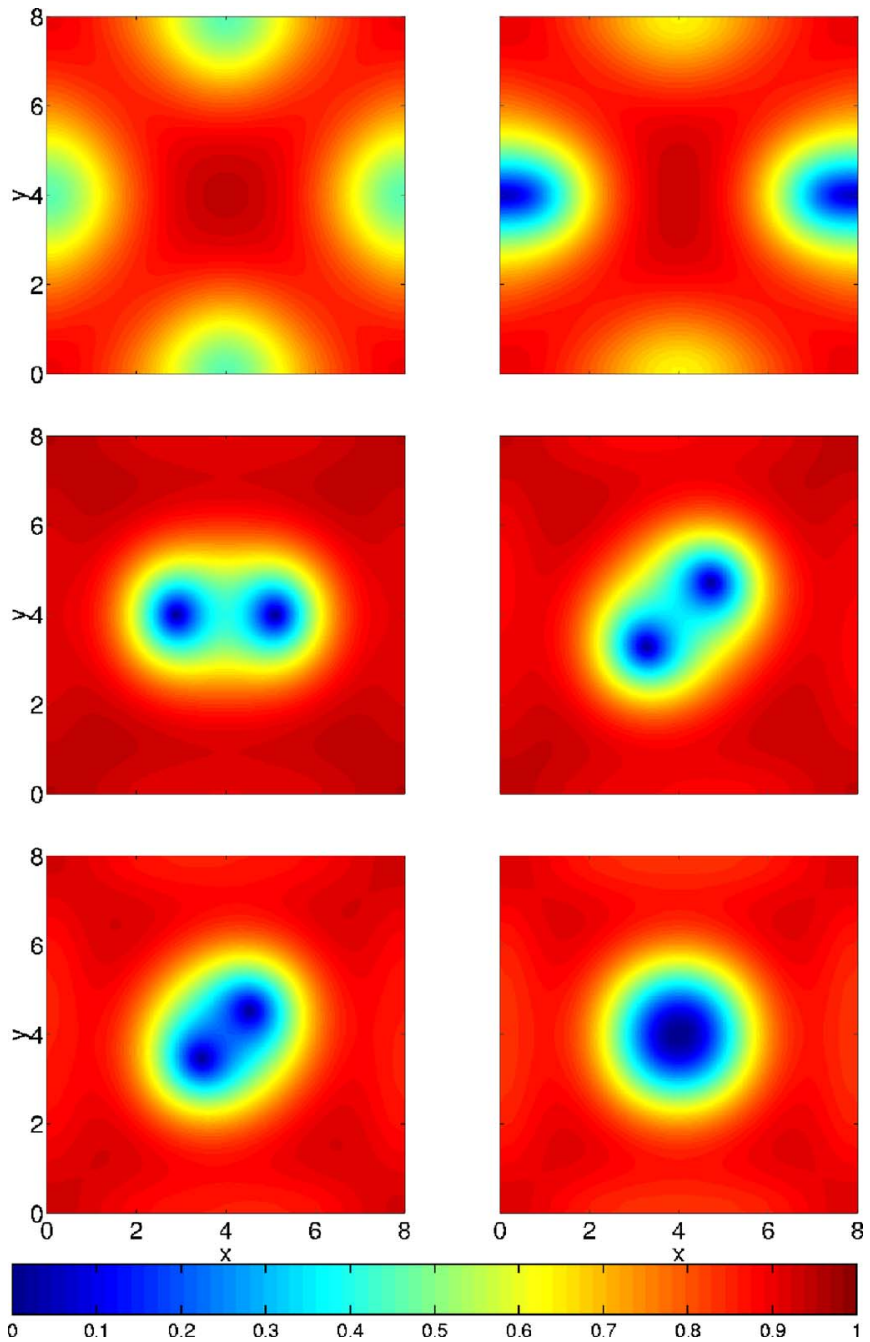

FIG. 4. (Color online) Sequence of pictures for the first phase transition in the $S_{1}$ superconductor at $T=0$; the sequence should be followed from the top to the bottom and from the left to the right. The colorbar indicates the magnitude of the order parameter.

\section{A. The upper critical field}

First of all, we calculated the upper critical field for the $S_{1}$ and $S_{2}$ superconductors. This result was useful in guiding us in which field window we had to work with in order to determine the other physical quantities. The criterion we have used for the total destruction of superconductivity is that at any $(i, j)$ vertex point and instant $n$ the magnitude of the order parameter $\left|\psi_{i, j}^{n}\right|<10^{-4}$. We slowly increased the applied magnetic field and kept track of the converged solution of the TDGL equations. Once the above criterion was fulfilled the corresponding values of the applied magnetic field was the upper critical field. The results are shown in Fig. 1. Notice that within the mesoscopic regime, the $H_{c 2}(T)$ curves do not change significantly with the surface area and they are both linear with respect to the temperature. To confirm that finite size effects are small we also have determined the upper critical field for $S_{3}=128 \times 128$ and $T=0.875$ and found no significant difference from $S_{2}$. Considering the linear dependence seen in Fig. 1, it is safe to affirm that the same is true for all temperatures. Figure 1 also shows the bulk values of $H_{c 2}(T)$, which is roughly half of its typical values for the systems we have studied. This tell us that, even though the mesoscopic behavior has been well characterized, it is still very distant from the thermodynamic limit. Since computational limitations preclude us from arbitrarily increasing system size, we were not able to determine the frontier between the two regimes.

\section{B. The energy and the magnetization}

On using Eqs. (13) and (14) we have evaluated the energy and the magnetization respectively, for several values of temperature ranging from $T=0$ to $T=0.875$. From now on we will restrict ourselves to the $S_{1}$ superconductor. The results for the magnetization and energy curves as functions of the applied external field are depicted in Fig. 2. As can be seen, the energy exhibits a series of discontinuities. Each of these discontinuities signals a vortex entrance. We attribute these abrupt reductions in the energy to flux expulsion. This point
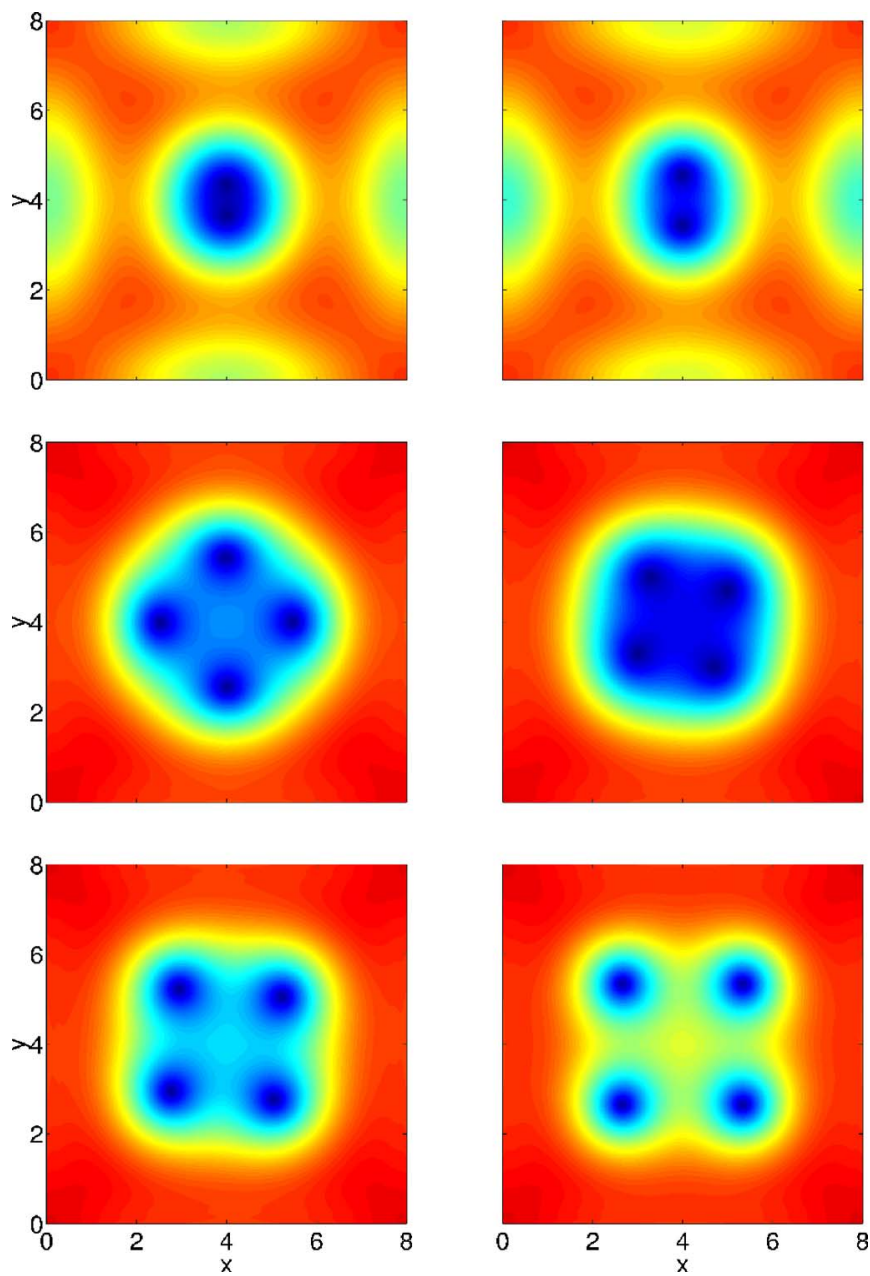

FIG. 5. (Color online) Sequence of pictures for the second phase transition in the $S_{1}$ superconductor at $T=0$; the sequence should be followed from the top to the bottom and from the left to the right. The magnitude of the order parameter is given by the colorbar of Fig. 4. 
TABLE I. The vortex configurations we have observed for each fixed temperature for the $S_{1}$ superconductor; the minus sign indicates that no configuration was observed with the respective vorticity. The nomenclature used is explained in the text.

\begin{tabular}{|c|c|c|c|c|c|c|c|c|}
\hline \multirow[b]{2}{*}{ Vorticity } & \multicolumn{8}{|c|}{ Temperature } \\
\hline & 0 & 0.125 & 0.25 & 0.375 & 0.5 & 0.625 & 0.75 & 0.875 \\
\hline 1 & - & - & - & - & - & - & $1 S$ & $1 S$ \\
\hline 2 & $2 S \rightarrow 1 G_{2}$ & $2 S \rightarrow 1 G_{2}$ & $1 G_{2}$ & $1 G_{2}$ & $1 G_{2}$ & $1 G_{2}$ & $1 G_{2}$ & - \\
\hline 3 & - & - & - & - & - & $1 G_{3}$ & - & - \\
\hline 4 & $4 S$ & $4 S$ & $4 S$ & $4 S$ & $4 S$ & $4 S$ & - & - \\
\hline 5 & - & - & $5 S$ & - & $5 S$ & - & - & - \\
\hline 6 & $4 S 1 G_{2}$ & $4 S 1 G_{2}$ & $4 S 1 G_{2}$ & $4 S 1 G_{2}$ & $4 S 1 G_{2}$ & - & - & - \\
\hline 7 & - & - & $4 S 1 G_{3}$ & $4 S 1 G_{3}$ & - & - & - & - \\
\hline 8 & $4 S 1 G_{4}$ & $4 S 1 G_{4}$ & $4 S 1 G_{4}$ & $4 S 1 G_{4}$ & - & - & - & - \\
\hline 9 & $4 S 1 G_{5}$ & - & $4 S 1 G_{5}$ & - & - & - & - & - \\
\hline 10 & $4 S 1 G_{6}$ & $4 S 1 G_{6}$ & $4 S 1 G_{6}$ & - & - & - & - & - \\
\hline 11 & $4 S 1 G_{7}$ & - & - & - & - & - & - & - \\
\hline 12 & $4 S 1 G_{8}$ & $4 S 1 G_{8}$ & - & - & - & - & - & - \\
\hline 13 & $4 S 1 G_{9}$ & - & - & - & - & - & - & - \\
\hline 14 & $4 S 1 G_{10}$ & - & - & - & - & - & - & - \\
\hline 15 & $4 S 1 G_{11}$ & - & - & - & - & - & - & - \\
\hline
\end{tabular}

will be better justified later on when we describe how the vortex motion occurs inside the sample.

We observed that for $T \leqslant 0.625$ the first vortex entrance always corresponds to a phase transition from $N=0$ to $N=2$ vortex state, and, similarly to the energy, the magnetization also decreases abruptly at the transition field. For $T \geqslant 0.75$ the energy also shows discontinuities as the applied field increases. Nevertheless, at the transitions the magnetization is continuous. In addition, only one vortex enters the sample at the first phase transition. This seems to be consistent with the fact that the dimension of the vortex tends to increase as we approach the critical temperature. So, there is less space available for the vortices to populate the sample. Notice that the field windows in which the magnetization is nonvanishing, vary with the temperature. At the zero temperature the system has the longest mixed state phase which is consistent with the phase diagram of Fig. 1. Furthermore, the magnetization does not scale with the temperature, that is, the behavior of this quantity is distinct for different temperatures.

For a fixed temperature, both the magnetization and the energy curves are composed of several field windows. Each window corresponds to a certain vorticity $N$. In Fig. 3 we illustrate the $N(H)$ curves calculated from Eq. (16) for lower temperatures; the inset of this figure shows the field window $\Delta H$ as a function of the vorticity $N$; here $\Delta H$ represents the amount of applied magnetic field required to take the system from $N$ to $N+2$ vortex state. As can be seen from these figures, the field window decreases as more vortices nucleate into the sample. However, $\Delta H$ has a weak dependence on the temperature.

The sequence of phase transitions does not follow the same pattern for different values of temperatures. In general, we have transitions either from $N$ to $N+2$, or from $N$ to $N$ +1 vortex state. This may vary for different temperatures or even within a fixed temperature. To describe an $N$ vortex state we use the following nomenclature. We denote by $N_{s} S$, a multiple vortex configuration formed by $N_{s}$ single vortices. A single giant vortex of vorticity $N_{g}$ is denoted by $1 G_{N_{g}}$; the constraint $N=N_{s}+N_{g}$ must be obeyed. For example, the $4 S 1 G_{2}$ state is formed by four single vortices and a double quantized giant vortex. All vortex configurations are summarized in Table I. Although our magnetization curves are very similar to others presented in the literature, ${ }^{1}$ it is necessary to emphasize that the simulations were performed considering the applied external field always increasing, and starting from the previous stationary state as the initial condition for the next value of $H$ (see Sec. II for a detailed explanation). Some differences can be noticed. It can be immediately seen that the $4 S$ state occurs for all temperatures $T \leqslant 0.625$. For $T$ not too close to the critical temperature, the $1 G_{2}$ state always exists. However, for lower temperatures $(T \leqslant 0.125)$, once two vortices penetrate the sample, first they form a $2 S$ state. Next, there is a smooth transition to a $1 G_{2}$ state. This transition is not manifested in the magnetization since there is no change in the vorticity, but only in the vortex arrangement. Notice that this particular scenario is highlighted in Table I. Also, the $1 S$ and $5 S$ states seem to occur less frequently. It can also be noticed that, different from other works, ${ }^{1}$ the $N$ $=2$ state is always observed as $1 G_{2}$. Another important result obtained in our simulations is that the $4 S$ state is observed to temperatures up to $T \leqslant 0.625$, without the evolution from MVS to GVS.

\section{The vortex configurations}

For all values of the applied magnetic field and between regular intervals of $n$, the magnitude of the order parameter and the local magnetic field were stored in matrices which were used to generate the time evolution of the vortex configurations. It must be stated that the sequence of figures 

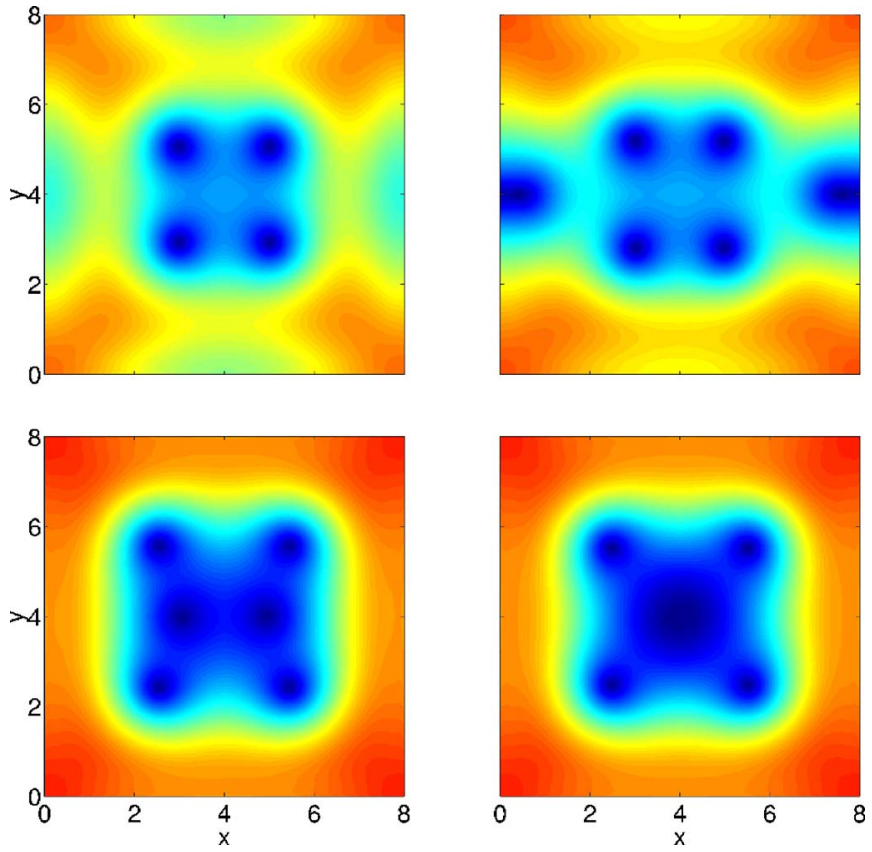

FIG. 6. (Color online) Sequence of pictures for the third phase transition in the $S_{1}$ superconductor at $T=0$; the sequence should be followed from the top to the bottom and from the left to the right. The magnitude of the order parameter is given by the colorbar of Fig. 4.

shown hereafter do not necessarily represent a collection of stationary states. To illustrate how the vortices evolve in time, first we chose the $T=0$ case. In Fig. 4, the phase transition from the Meissner to the $N=2$ state is presented. First, there are four vortices trying to nucleate at the surfaces, but only two will succeed to enter the sample, and the other two are driven out. This explains the reduction in the value of the free energy as we have stressed previously. Once the two vortices have completely penetrated the sample, they form a $2 S$ state aligned as shown in Fig. 4. Next, they will try to align along the square diagonal. Since this configuration is double degenerated, they stay in a significant range of applied magnetic field aligned perpendicular to the vertical sides of the square, before they go to the square diagonal. As the applied external field increases, the vortices will slowly rotate until they are along the square diagonal. Such a result was also found by Mel'nikov et al. ${ }^{7}$ In their work, the vortices also accomodate in the diagonal configuration. However, since we follow the time evolution, we were able to notice that the $2 S$ configuration collapses into a $1 G_{2}$ state. This also occurs for $T=0.125$, although in a smaller field window. Otherwise, for the values of $0.25 \leqslant T \leqslant 0.625$, the $1 G_{2}$ state is formed straightway, that is, no additional applied field is required to collapse into this state after the phase transition occurs.

In opposition to the situation described above, all the phase transitions we sketch below occurs at a fixed applied magnetic field.

Now we discuss the phase transition $1 G_{2} \rightarrow 4 S$, which occurs for a wide range of temperature (see Fig. 5). Initially, there are four vortices trying to nucleate at the surface. As in the previous case described above, only two vortices can
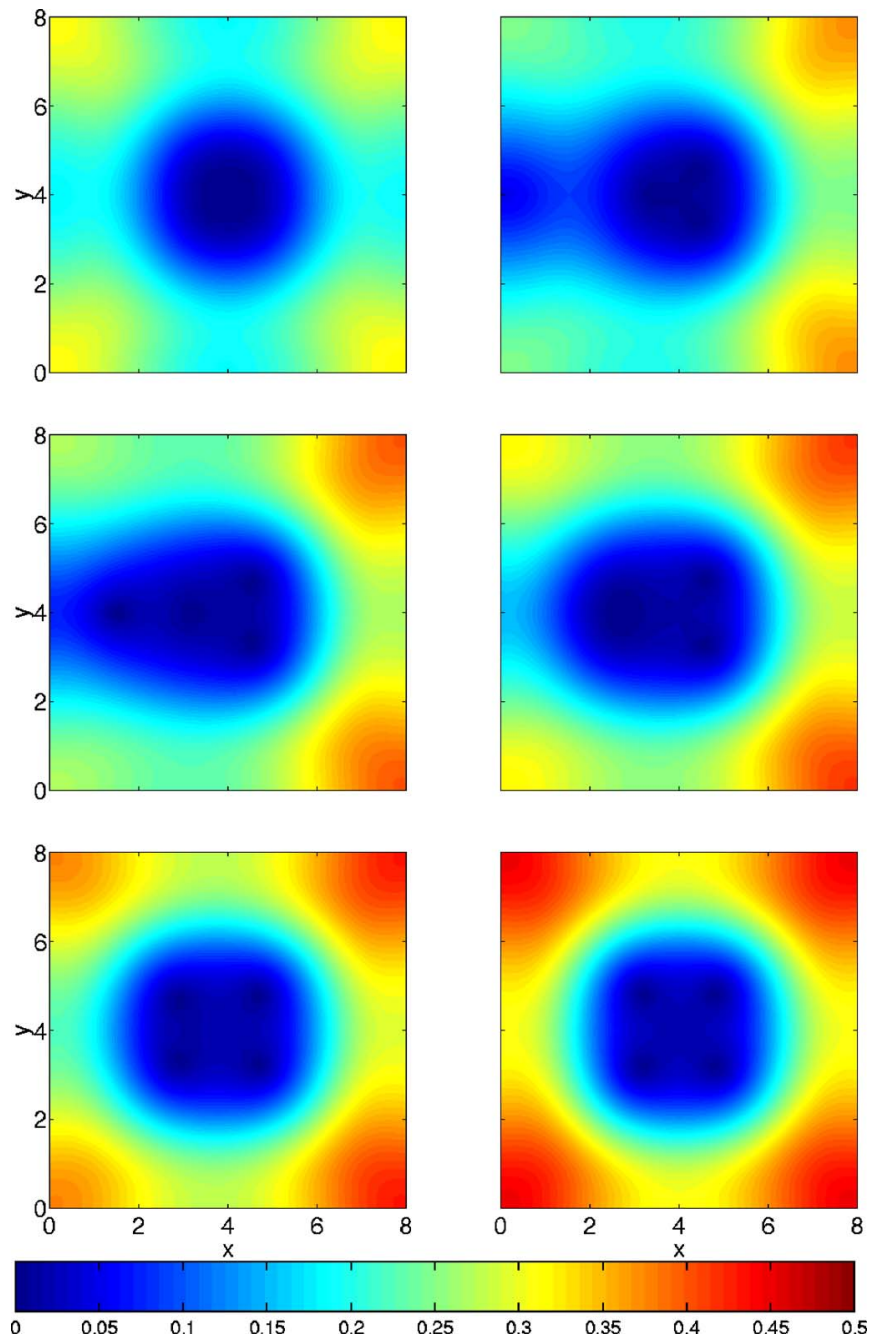

FIG. 7. (Color online) Sequence of pictures for the second phase transition in the $S_{1}$ square superconductor at $T=0.625$; the sequence should be followed from the top to the bottom and from the left to the right. The colorbar indicates the magnitude of the order parameter.

enter the sample. While these two vortices are moving toward the center, the giant vortex in the center is breaking off. Just after the two new vortices have completed penetrating the sample, the $4 S$ state is created forming a square. Then, they will rotate in order to fit into the sample. We have found that this rotation occurs very rapidly until the $4 S$ state stabilizes. Notice that while the $4 S$ square is rotating, the vortices keep moving forward and then backward.

Let us show how the phase transition $4 S \rightarrow 4 S 1 G_{2}$ takes place (see Fig. 6). Similar to the previous situations, among four vortices, two move back and the other two enter the sample. The two new vortices will pass through the $4 S$ square and collapse at the center of the superconducting square and, consequently, a $1 G_{2}$ state is formed.

As can be seen from Table I, the state $3 S$ was not observed. This in agreement with the work of Mel'nikov et al. In fact, they constructed the state $N=3$, either considering a $4 S$ state plus an antivortex at the center of the square or a single $3 S$ state with a broken symmetry caused by a defect. 
Finally, it is interesting to describe a quite unique phase transition $1 G_{3} \rightarrow 4 S$, which happens only at $T=0.625$ (see Fig. 7). In this case, only one vortex is able to enter the sample. Once it is inside the superconductor, the $1 G_{3}$ state will break off into a trianglelike $3 S$ state. The inner vortex of the $3 S$ state collides with the penetrated vortex, coming from the left-hand side of the square superconductor. Just after the collision, they move to the vertices of a square $(4 S)$ which fits into the sample.

In Ref. 12 we have provided animations which describe the vortex motions from the beginning of the Meissner phase until the superconductivity is completely suppressed for all temperatures.

\section{SUMMARY}

We have solved numerically the TDGL equations for a square mesoscopic superconductor by using the $\psi-U$ method.
Our implementation of the algorithm provides all possible configurations of the vortices for a wide range of temperatures. Furthermore, our approach makes it possible to observe the time evolution of the vortex configurations, that is, we have been able to see how GV and MV states are formed and how the transitions from GV into MV states (and vice versa) may occur. We also have demonstrated that the temperature has a crucial role in the formation of these states. In spite of some slight differences, our results agree well with previous works. ${ }^{1,6}$ Perhaps these differences may be attributed to the different geometry and the parameters used.

\section{ACKNOWLEDGMENTS}

The authors thank the Brazilian Agencies FAPESP and CNPq for financial support.
*Electronic address: sardella@fc.unesp.br

†Electronic address: malvezzi@fc.unesp.br

†Electronic address: plisboa@fc.unesp.br

§Electronic address: wortiz@df.ufscar.br

${ }^{1}$ B. J. Baelus and F. M. Peeters, Phys. Rev. B 65, 104515 (2002).

${ }^{2}$ M. Morelle, J. Bekaert, and V. V. Moshchalkov, Phys. Rev. B 70, 094503 (2004).

${ }^{3}$ V. A. Schweigert, F. M. Peeters, and P. S. Deo, Phys. Rev. Lett. 81, 2783 (1998).

${ }^{4}$ A. Kanda, B. J. Baelus, F. M. Peeters, K. Kadowaki, and Y. Ootuka, Phys. Rev. Lett. 93, 257002 (2004).

${ }^{5}$ S. Okayasu, T. Nishio, Y. Hata, J. Suzuki, I. Kakeya, K. Kadowaki, and V. Moschchalkov, IEEE Trans. Appl. Supercond. 15, 678 (2005).
${ }^{6}$ B. J. Baelus, A. Kanda, N. Shimizu, K. Tadano, Y. Ootuka, K. Kadowaki, and F. M. Peeters, Phys. Rev. B 73, 024514 (2006).

${ }^{7}$ A. S. Mel'nikov, I. M. Nefedov, D. A. Ryzhov, I. A. Shereshevskii, V. M. Vinokur, and P. P. Vysheslavtsev, Phys. Rev. B 65, 140503(R) (2002).

${ }^{8}$ A. D. Hernández, B. J. Baelus, D. Domínguez, and F. M. Peeters, Phys. Rev. B 71, 214524 (2005).

${ }^{9}$ A. Schmid, Phys. Kondens. Mater. 5, 302 (1966).

${ }^{10}$ W. D. Gropp, H. G. Kaper, G. K. Leaf, D. M. Levine, M. Palumbo, and V. M. Vinokur, J. Comput. Phys. 123, 254 (1996).

${ }^{11}$ G. C. Buscaglia, C. Bolech, and A. López, in Connectivity and Superconductivity, edited by J. Berger and J. Rubinstein (Springer, Berlin, 2000).

${ }^{12}$ See http://www.fc.unesp.br/supercondutores 